\title{
Effects of polyphenol rich bamboo leaf on rumen fermentation characteristics and methane gas production in an in vitro condition
}

\begin{abstract}
The aim of this study was to test the effect of bamboo leaf (BL) on rumen methane gas production and rumen fermentation characteristics, in vitro. Different amounts of BL; CON (0\%), Low BL (LBL, $10 \%$ ), Medium BL (MBL, 15\%) and High BL (HBL, 25\%) of replacement with alfalfa hay $(\mathrm{AH})$ in substrate $(50 \%$ concentrate $+50 \% \mathrm{AH})$ were mixed with 30 millilitre $(\mathrm{mL})$ of buffered rumen liquor for $48 \mathrm{~h}$ of incubation. Total gas production $(\mathrm{mL} / 250 \mathrm{mg} \mathrm{DM})$ was not affected $(\mathrm{P}>0.05)$ among BL treatment groups at different times of incubation. Production of methane gas $(\mathrm{mL} / 250 \mathrm{mg} \mathrm{DM})$ decreased at a declining rate $(\mathrm{P}<0.05)$ with higher $\mathrm{BL}$ levels. Methane gas inhibitory effects of BL treatment groups as compared with CON were; $29 \%, 35 \%$ and $62 \%$ for LBL, MBL and HBL, respectively. The ratio of acetic/propionic was lowest $(\mathrm{P}<0.05)$ for HBL (1.67) as compared to CON (2.09).
\end{abstract}

Keyword: Bamboo leaf; Fermentation; In vitro gas production; Methanogenesis; Rumen 